\title{
Can nutrition counselling be more behavioural? Lessons learned from dietary management of cystic fibrosis
}

\author{
Lori J. Stark \\ Cincinnati Children's Hospital Medical Centre and University of Cincinnati College of Medicine, \\ Division of Psychology, MLC D3015, 3333 Burnet Avenue, Cincinnati, OH 45229, USA
}

\begin{abstract}
Cystic fibrosis (CF) is a genetically-inherited disorder that results in energy imbalance. Undernutrition is common in children with $\mathrm{CF}$ and associated with poor health outcomes. To ensure optimal growth and nutrition, children with CF are recommended to consume 120-150\% of the recommended daily allowance (RDA) for energy, but most studies show they typically are only able to achieve $100 \%$ of the RDA. While biological factors clearly contribute to poor dietary adherence, recent studies have documented behavioural and environmental barriers to adherence that includes parent-child interaction at mealtimes. While not 'abnormal', parent behaviours such as paying increased attention to the child in the form of coaxing, commanding and feeding when the child is engaged in behaviours incompatible with eating (food refusal, talking, leaving the meal) may serve to reinforce these child non-eating behaviours. Thus, dietary counselling alone, albeit necessary, is typically insufficient because of failure to specifically address these behavioural and environmental barriers to dietary treatment. Behavioural intervention that targets both nutrition education and behavioural management has been found to be effective in achieving an average increased energy intake of $4200 \mathrm{~kJ}(1000 \mathrm{kcal}) / \mathrm{d}$ and weight gain of $1.48 \mathrm{~kg}$ over 9 weeks in children with CF. This intervention utilizes self-monitoring, goal setting and shaping to structure the delivery of treatment. It also teaches parents to utilize child behaviour-management techniques to motivate children to increase their energy intake. These behavioural strategies include differential attention (praising and ignoring), contingency management and behavioural contracting. The potential application of these techniques to dietary counselling is suggested.
\end{abstract}

Cystic fibrosis: Behavioural intervention: Nutrition counselling: Weight gain:

Energy intake

\section{Background: importance of nutrition in cystic fibrosis}

Cystic fibrosis (CF) is a genetically-transmitted disease that affects the exocrine or secretory glands of several major organs in the respiratory, gastrointestinal and reproductive systems. The most detrimental effects of the disease are to the lungs and pancreas. While $90 \%$ of deaths are due to chronic progressive pulmonary disease, which results in early death due to cardio-respiratory failure or complications, nutritional status plays a key role in survival and health (Cystic Fibrosis Foundation, 2002). Poor nutritional status is associated with poor survival (Corey \& Farewell, 1996) and poor pulmonary functioning (Zemel et al. 2000; Beker et al. 2001). Nutritional status is compromised in patients with $\mathrm{CF}$ because of the energy imbalance caused by increased energy requirements of the chronic lung disease, acute pulmonary infections and energy loss secondary to malabsorption (Ramsey et al. 1992). To offset this energy demand patients with $\mathrm{CF}$ are recommended to increase their energy intake to $120-150 \%$ of the recommended daily allowance (RDA) of energy for individuals without $\mathrm{CF}$ (Borowitz et al. 2002).

\section{History of dietary recommendations and adherence}

Before the 1980 s patients with $\mathrm{CF}$ were recommended to consume $120-150 \%$ of the RDA for energy on a low-fat diet. A low-fat diet was hypothesized to control symptoms of stomach cramping and diarrhoea. In addition, malabsorption of fat was thought to interfere with absorption of 
other nutrients (Crozier, 1974). Adherence to the CF dietary recommendations was nearly impossible on the low-fat diet. Several studies conducted during this time demonstrated that not only were patients with CF not achieving the CF dietary recommendations, they were below RDA for healthy individuals and were achieving only $80 \%$ of the RDA for energy (Chase et al. 1979; Smith \& Lloyd-Still, 1983).

In 1973 the Toronto CF Center (Toronto, Ont., Canada) changed their approach to dietary management of $\mathrm{CF}$ and recommended a diet high in saturated fat with emphasis on whole milk, butter and animal fat, and reported an immediate improvement in body weight for patients (Crozier, 1974). The benefits of a high-fat diet were not recognized until the 1980 s when dietary recommendations for patients with $\mathrm{CF}$ were changed. In a seminal paper comparing the nutritional status and survival of CF patients prescribed a high-fat diet at the Toronto CF Center with CF patients treated on a low-fat diet at a CF centre in Boston, MA, USA, Corey et al. (1988) reported that the Toronto patients of all ages exhibited higher weight percentiles than the Boston patients. They also found a greater median age of survival for the Toronto patients (30 years) compared with the Boston patients (21 years).

The change from a low-fat diet to a high-fat diet made it easier for patients to achieve a higher percentage RDA for energy, but the change did not necessarily help patients with CF achieve their dietary recommendations. In Australia Daniels et al. (1987) compared the percentage RDA for energy of patients with CF aged 7 months -12 years before and after the implementation of the non-restricted-fat diet. While they found that the children with CF demonstrated a greater percentage RDA for energy on the non-restricted-fat diet than on the fat-restricted diet, only $39 \%$ of the patients achieved the CF dietary recommendation of $120 \%$ of the RDA for energy. In subsequent studies in the USA and UK children with $\mathrm{CF}$ aged 7 months -12 years have been found to be consuming approximately $100 \%$ of the RDA for energy, with just a small percentage (11-23) of any sample found to be achieving $120 \%$ of the RDA for energy (Tomezsko et al. 1992; Stark et al. 1995; Anthony et al. 1999; Powers et al. 2002). Clearly, the change to a high-fat diet has been beneficial in the treatment of CF; however, it has been insufficient to attain the dietary recommendations for CF.

Early intervention efforts with patients with CF targeted improving education about diet. These studies found an increase in dietary knowledge, but no improvement in energy intake (Bell et al. 1984). The health care team in most US CF centres includes a dietitian who provides dietary counselling on a routine basis for patients attending the centre. The definition of routine dietary counselling, however, varies widely across centres and is affected by financial support for such services, the number of dietitians relative to the number of patients, and the period of time that the dietitian can dedicate to CF care. Many families of children with CF may only have contact with a dietitian once yearly at their annual visit, with more intensive contact available only for those children failing to gain weight, or who are actually losing weight. However, even 'intensive contact' may be only once every 3 months at the patient's routine $\mathrm{CF}$ centre appointment. The inconsistent availability of dietary counselling may account for the findings of many studies that parents of children with CF report their child has no special dietary recommendations (Henley \& Hill, 1990; Eddy et al. 1998).

\section{Barriers to dietary adherence for children with cystic fibrosis}

Bowen \& Stark (1991) published a review paper on malnutrition in CF and postulated behavioural and environmental barriers to dietary treatment adherence. While it was acknowledged that biological factors such as food palatability and satiety may inhibit dietary adherence in $\mathrm{CF}$, it was also noted that feeding and eating occur within the context of parent and child interactions and socialization. Within this context, parental mismanagement of child eating behaviour, such as attention to non-eating in the form of parental coaxing or making a second meal, may serve to reinforce non-eating behaviour in children with $\mathrm{CF}$. Parent reports of mealtime problems for children with CF aged 1-7 years support this hypothesis (Crist et al. 1994). Crist et al. (1994) found that parents of children with $\mathrm{CF}$ regarded mealtime issues such as meals lasting $>20 \mathrm{~min}$, children dawdling, refusing and spitting out food, having a poor appetite and choking or gagging as more problematic than parents of same-age children without CF. They also found that parents of children with $\mathrm{CF}$ engaged in more problematic mealtime management strategies, such as using threats, force feeding, getting angry with their child and coaxing, more often than parents of children without CF. Parents of children with CF also reported less confidence in their ability to feed their child than parents of controls. Finally Crist et al. (1994) found a negative correlation between the parent report of child mealtime problems and energy intake within the CF sample.

Hypothesizing that children with $\mathrm{CF}$ engage in maladaptive eating behaviours and that their parents exhibit behavioural mismanagement of child behaviour during mealtimes (Bowen \& Stark, 1991), a series of observational studies was conducted comparing children with $\mathrm{CF}$ and their families with children without $\mathrm{CF}$ and their families across three developmental periods: infant and toddler, preschool and school-age. In the study of preschool children (Stark et al.2000) it was found that children with CF and children without CF took fewer bites and engaged in behaviours incompatible with eating more frequently during the second half of the meal than they did during the first half of the meal. Children with $\mathrm{CF}$, however, engaged in these behaviours more often than controls. As children lost interest in eating during the second half of the meal, parents demonstrated an increase in their behaviours to encourage eating by giving more commands to eat, coaxing, feeding the child and using physical prompts (Stark et al. 2000). In fact, parents of both groups of children engaged in remarkably similar parenting strategies to encourage eating, but parents of children with $\mathrm{CF}$ used these strategies more frequently at meals than parents of controls. In addition, parents of children with CF kept their child at the meal an average of 6 min longer than did parents of controls (Stark et al. 1995). A similar pattern emerged for the infant and toddler and school-age periods. As early as at 6-36 months parents of 
children with CF have been shown to keep their child at the meal an average of 4 min longer than parents of children without CF (Powers et al. 2002). This pattern extended into school age, when meals of children with CF were an average of 7 min longer than those of controls (Stark et al. 1997). From the results of these studies it was concluded that dietary recommendations for the young patient with $\mathrm{CF}$ to 'eat more' results in parents attempting to encourage eating. The parents of children with CF do not adopt different maladaptive strategies from parents of children without $\mathrm{CF}$ to encourage eating. Instead, they appear to escalate their use of typical parenting strategies that are not effective for any child (Satter, 1987) regardless of health status. These strategies typically involve increased parental attention to the child when they are not eating. Behavioural theory suggests that by following a behaviour with a desirable outcome the likelihood of that behaviour occurring in the future is increased. The desirable outcome in the case of children with CF is the receipt of increased parental attention. Thus, based on behavioural theory, parents may be reinforcing 'not eating' by increasing their attention to their child when the child is engaged in behaviours incompatible with eating. For children without specific dietary recommendations, such as is the case for the controls, parental attempts to encourage eating are minimal in frequency and in period of time spent at the meal. Thus, in this sample, as well as in well-nourished healthy children in general, these parental behaviours do not typically result in food struggles. After all, the parent of a well-nourished child can rationalize that their child is growing fine. Thus, eating less food at one meal will not compromise his or her overall health. In contrast, for the parent of a child with CF one more bite is always better and one step closer to optimum health.

Whereas children with CF and their parents do not differ from controls in the types of behaviours exhibited at meals, subsequent analysis of the preschool sample revealed that the increased frequency of parent and child behaviours has a effect on family functioning at mealtimes (Spieth et al. 2001). Families of preschool children with CF scored lower on six of seven domains of family functioning and in the 'unhealthy' range for all seven domains of family functioning in comparison with families of children without $\mathrm{CF}$, who scored in the 'healthy' range. These findings indicated that commonly-used parenting strategies may have negative effects on family functioning at meals when used at a high frequency. In a study by Sanders et al. (1997) in Australia mothers of children with CF were found to perceive their child's mealtime behaviours as more problematic and were observed to use a higher frequency of aversive behaviours and lower levels of positive behaviours to manage the meal than control mothers. Of particular note, and similar to the results reported by Crist et al. (1994), parents of children with $\mathrm{CF}$ reported lower ratings of parenting self-efficacy than parents of children diagnosed with feeding problems and parents of children without a health condition.

\section{Behavioural treatment to improve energy intake in children with cystic fibrosis}

From our assessment studies, provision of nutritional recommendations to increase the food intake of a child with
CF appears to be necessary, but insufficient to meet $\mathrm{CF}$ dietary recommendations. When equipped with the knowledge that children with CF need to have a higher energy intake, parents respond by escalating their use of typical parenting strategies of coaxing, prompting, commanding and feeding of young children (Stark et al. 2000). While typical, these strategies do not result in an increased energy intake that meets the CF dietary recommendations (Stark et al. 1995) and may be counterproductive (Spieth et al. 2001). Thus, it was believed that an effective intervention would need to provide parents with alternative evidenced-based child management strategies to change child eating behaviour. In addition, adherence to medical regimens in general has been found to decrease as the complexity of the treatment regimen and duration of the disease increases (Meichenbaum \& Turk, 1987). Dietary treatment is complex and long term. Thus, an intervention strategy was developed that addressed the behavioural and environmental barriers identified in observational studies by incorporating behavioural strategies to promote long-term change in eating and reducing the complexity of dietary adherence.

The behavioural intervention, Behavioural Intervention for Change Around Growth and Energy (Be In CHARGE!), is a group treatment that targets children with $\mathrm{CF}$ aged 4-12 years and their parents. Children and parents are seen simultaneously, but in their own parent or child group. The parent group is given nutritional education plus training in child behavioural management. This training is conducted in a systematic step-by-step fashion that targets one meal and one child behaviour management strategy at each session. Parents monitor their child's food and energy intakes daily throughout treatment and are provided feedback on their child's intake via graphs showing energy intake and energy goals. An increase of $1050 \mathrm{~kJ}(250 \mathrm{kcal}) / \mathrm{d}$ is targeted for each meal. Suggestions for achieving this increase in energy intake are provided in general written handouts and through individualized handouts tailored to the child's typical food intake. Treatment typically targeted snacks first because it is considered the easiest meal to change, as parents are not optimizing the number of snacks possible each day or the energy density of the foods served. In addition, a snack is typically not as 'emotionally charged' as other meals such as dinner where time and energy is put into the meal preparation.

The child behavioural-management strategies taught are extracted from evidenced-based interventions for general child behavioural problems (Forehand \& McMahon, 1981) and treatments for children with feeding problems (Linscheid, 1983; Linscheid et al. 1995) and obesity (Epstein et al. 1980). Specifically, child behaviours that lead to increased parental attention are likely to persist and even increase, while child behaviours that do not receive parental attention tend to decrease. From our observational data, parents typically increased their attention to their child in the form of coaxing, commanding, physically prompting and even feeding their child when the child was not eating. In addition, despite parental perception that their children did not eat, children with $\mathrm{CF}$ did eat and ate an amount comparable with that of a child without CF. Parents, however, rarely noticed appropriate eating. Thus, in the 
intervention parents are taught initially to notice and pay attention to their child when the child is eating. Parents are given examples of how to specifically describe what they like about the child's behaviour (e.g. 'Jimmy, I liked the way you got started on your snack right away!') and to compliment the child in age-appropriate and meaningful ways. Parents and children are also provided with a sticker chart for the parents to use to help their child track their success in meeting their 'energy goals' at each meal and day. When a child meets their 'energy goal' at a meal at home, parents are instructed to place a sticker on the chart and compliment the child.

While powerful, praise alone will not change behaviour if attention is also given equally for undesirable behaviour. Thus, parents are taught to identify and ignore behaviours incompatible with eating such as excess talking, dawdling, playing with food and complaints about food or amount of food served. These skills are taught via didactics, written handouts and role plays in which parents identify the most challenging child mealtime behaviours in their families and rehearse use of the praising and ignoring skills in the treatment group. Parents are then taught to identify important mealtime rules for their family that will promote optimal eating. For example, if a child frequently leaves the table, a family may choose a rule enforcing the child to stay seated until the parents excuse him or her. As children with $\mathrm{CF}$ tend to have long meals and the literature on satiety indicates that individuals tend to feel full after $20 \mathrm{~min}$ of eating, parents are encouraged to set a time limit of $20 \mathrm{~min}$ for meals and establish a mealtime rule that children must meet their 'energy' goal within $20 \mathrm{~min}$. Parents are then taught how to identify and implement appropriate homebased rewards for their child meeting their energy goals and following mealtime rules. Behavioural contracting is used to explicitly define the expected child behaviour and the reward that parents will provide. Finally, parents are taught how to combine the child management strategies and use 'shaping' to encourage children to eat a variety of foods and try novel foods. Shaping refers to a procedure in which a behaviour is broken down into its component steps, with the most basic taught first. In the case of new foods parents are encouraged to start gradually introducing a small amount of a new food on the child's plate, without expectations that the child eats it. As the child becomes accustomed to the presence of the food, parents then increase the required behaviour from the child tolerating it on their plate, to tasting it, or taking a bite. Throughout this process parents learn about research findings on food preferences that suggests that several exposures are necessary for a food to become familiar and liked (Birch, 1990).

In the child group the child management strategies taught to the parents are used to conduct the group. Children are praised for behaviours compatible with participating in the group and minor disruptive behaviours (talking out of turn) are ignored. Children are provided with the rationale for the group in developmentally-appropriate language that discusses the importance of energy for growth and play, and teaches that energy comes from food. Each week the children engage in a fun activity designed to teach them about high-energy foods for the meal targeted. The children are also provided a practice meal when they are taught to increase the energy of typical foods and are rewarded for meeting their meal energy goal in the group session. As described earlier, children are given a sticker chart each week to track their progress with meeting their energy goal at home for each meal and each day. Only one new meal is targeted at each session and children are awarded a trophy in the session when they earn five of seven daily energy goal stickers over 1 week.

Treatment studies conducted over the past 12 years indicate that $\mathrm{Be}$ In CHARGE! is highly efficacious. In two studies children with CF served as their own controls. Energy intake increased by an average of $3780-4200 \mathrm{~kJ}$ $(900-1000 \mathrm{kcal}) / \mathrm{d}$ pre- to post-treatment and weight gains of 0.66-1.48 kg were achieved (Stark et al. 1990, 1993). A third study demonstrated that Be In CHARGE! was more efficacious than standard of care. Children receiving Be In CHARGE! increased their energy intake by approximately $4200 \mathrm{~kJ}(1000 \mathrm{kcal}) / \mathrm{d}$ and had a weight gain of $1.7 \mathrm{~kg}$, while children in a wait-list control group showed a minimal change in energy intake $(1025 \mathrm{~kJ}(244 \mathrm{kcal}) / \mathrm{d})$ and no change in weight (Stark et al. 1996). In a recent pilot study Be In CHARGE! was compared with a nutrition education programme that had the same time commitment, contact with health care professionals and nutritional information. While the sample size was too small to detect significance, children participating in Be In CHARGE! increased their energy intake by $4351 \mathrm{~kJ}(1036 \mathrm{kcal}) / \mathrm{d}$ and gained $1.42 \mathrm{~kg}$ over 9 weeks, while for the children receiving the nutrition education the increases were only half those amounts, energy intake $1714 \mathrm{~kJ}(408 \mathrm{kcal}) / \mathrm{d}$ and weight gain $0.78 \mathrm{~kg}$ (Stark et al. 2003).

It is of clinical importance that although the combination of behavioural intervention and nutritional education was almost twice as effective as nutrition education alone, the nutrition education intervention was more effective over the short period of intervention (9 weeks) than previously-reported education-only interventions (Bell et al. 1984) and standard of care (Stark et al. 1996). It is hypothesized that the unexpected improvement in energy intake and weight gain found with the nutrition education intervention in this study was due to the incorporation of many behavioural strategies in the structure of the intervention. The structure was distinctly different from the manner in which nutritional education is typically provided and may have implications for improving the efficacy of nutritional counselling. Indeed, Luder et al. (1989), utilizing a systematic and tailored approach to nutritional counselling for patients with $\mathrm{CF}$, found an increased energy intake to the recommended treatment level of $120 \%$ of the RDA for energy within 3 years of introducing this method to dietary counselling. In this study thirty-seven patients with CF ranging in age from 2 years to 27 years were seen for nutritional counselling every 3 months. Individualized meal plans were tailored to the patient's food preferences, and goals were set based on the patient's individual energy needs and environmental support. Adherence was monitored via $24 \mathrm{~h}$ dietary recall. When patients did not meet dietary goals, the dietitian problem solved barriers to the dietary recommendations and made changes in the plan. Thus, although developing a systematic approach to dietary counselling appears beneficial, a combined approach that 
incorporates behaviour-change strategies that address specific barriers such as problematic parent-child interactions together with nutritional counselling appears to hold greater promise for improving adherence to dietary changes. In addition, this combined approach appears to have a greater effect on adherence than nutrition information alone, regardless of how nutrition counselling is presented.

\section{Making nutrition counselling more behavioural}

From the results of our research it appears that several behavioural strategies could be easily incorporated into dietary counselling and may increase the effectiveness of dietary counselling alone. These strategies included systematic use of self-monitoring, graphic feedback of progress based on self-monitoring, setting goals that gradually increase in difficulty and tailoring treatment recommendations. Each of these strategies will be discussed.

\section{Self-monitoring}

This strategy has been shown to be effective in modifying behaviour, at least in the short term (La Greca \& Schuman, 1995). In the author's research ongoing monitoring of dietary intake is an instrumental teaching tool that provides opportunities for setting short- and long-term dietary goals, identifying barriers to dietary change, defining solutions to these barriers and rewarding children with $\mathrm{CF}$ and their parents for meeting their energy goals. For example, if weekends are consistently found as a time during which children cannot meet their energy goal, the dietary monitoring allows problem solving to determine if the failure to meet the goal was due to lack of time, lack of structure or the child being too busy doing other activities. Once the barrier is identified, the families can be worked with to generate solutions to overcome these barriers.

\section{Feedback}

Feedback on self-report data is critical. Diet diaries are timeconsuming and labour intensive for parents to complete and, therefore, feedback based on these diet diaries is necessary for motivation as well as behaviour change. Provision of visual feedback in the form of graphs clearly demonstrates changes individuals have made in diet. Again, these data are important in assessing barriers and progress. In the author's work with $\mathrm{CF}$, parents always feel pressure to increase their child's food intake and rarely get the feedback that they have accomplished enough, or even receive information on how close they are to achieving a goal. To be most effective, feedback must focus on the positive changes, regardless of the magnitude of the change. Moreover, barriers to achieving a goal need to be assessed in an empathetic and supportive manner.

\section{Setting goals and shaping}

These processes go together and are important to success. To be effective, goals initially should be set so that they are obtainable and gradually increased until the ultimate goal is accomplished. This process is referred to as 'shaping'.
In many health conditions the need for dietary change is immediate. Dietary patterns, however, are learned behaviours that have been acquired and reinforced over a long period of time. Changing all aspects of an individual's eating patterns at once is difficult, if not impossible. Thus, breaking the process down into a succession of steps that eventually achieves the final 'dietary goal' and helping patients to focus on changes in one area at a time is likely to be more successful than giving parents and patients all the information at once and leaving the 'how to' and the sequence of the implementation of dietary change to the patient. In the author's CF research children and parents target change at only one meal at a time. This 'shaping' strategy allows them to build up the confidence in their ability to change behaviour as they build up their skills at making these changes.

\section{Tailored interventions}

These interventions are more effective than generic interventions (Ryan \& Lauver, 2002). In the author's research families of children with $\mathrm{CF}$ are given general guidelines for increasing energy intake at a given meal. In addition, they are given individualized tailored handouts that explicitly discuss ways of incorporating the general guidelines into their child's food intake. The individual presentation of the general guidelines allows parents to easily identify ways of incorporating energy guidelines from the handouts into their child's diet.

\section{Behavioural intervention}

In addition to applying behavioural principles to the structure of the intervention, it is likely that behavioural treatments may need to be applied when working with patients and families. These strategies included identification of barriers to dietary changes, reinforcement, contingency management, self-reward and contracting.

\section{Identify barriers to treatment}

In the author's research with CF patients, specific barriers to achieving adherence to dietary recommendations have been identified. A common barrier was ineffective parentchild interactions during mealtimes, a challenge that has been specifically addressed in the dietary intervention. Thus, the cornerstone of the intervention is child behaviourmanagement training, in which parents are taught the principles of behaviour management and the manner in which to implement these principles during mealtimes with their children. In other clinical populations with dietary recommendations other barriers may be more salient and should be assessed to identify the most efficacious intervention. Self-monitoring of the target behaviour provides one such avenue of assessment. By reviewing the self-monitoring data, the range of the behaviour and how much or how little energy is consumed across a specific period of time can be assessed. Self-monitoring data also allows the identification of days or meals that are most problematic for adherence and the barriers to adherence for those days or meals. 


\section{Reinforcement}

Behaviours are typically maintained by the consequences that follow them. In the case of dietary adherence and children with $\mathrm{CF}$ it is hypothesized that while there are numerous biological factors that contribute to eating and satiety in CF, parent attention to child behaviours incompatible with eating are inadvertently reinforcing child behaviours such as dawdling, food refusal and leaving the table. It is also hypothesized that child eating is typically not attended to, because the children are doing 'what they are suppose to do' and thus parents are less likely to notice or attend to these behaviours. Based on these observations, the parents are worked with to change the consequences of eating and not eating such that eating results in parental attention and compliments and behaviours incompatible with eating are ignored by parents. This technique is referred to as differential attention and defined as the awarding and withholding of attention contingent upon the emission of a desired behaviour.

\section{Contingency management}

Eating behaviours are often difficult to change for children (and adults). Thus, it has been found to be necessary to use contingency management in addition to differential attention. The approach is to problem solve with parents the type of rewards that the children can earn on a daily basis for achieving their energy goals. These rewards include privileges such as access to computer time or special time with a parent. Again, it is important to identify a privilege that has value to the child. Simultaneously, it is necessary to ensure that the child's attainment of the privilege is not one that is more important to the parent (e.g. sharing of a bedtime story) than the child and that the privilege is not an expensive object or activity that cannot be awarded routinely. These privileges are then given on a daily and weekly basis. Identifying appropriate rewards and consequences for behaviour change is critical when teaching or introducing a new behaviour. Just as critical to the success and behaviour change is the notion that, when first initiating a new behaviour, parents must give the reward close in time to the desired behaviour change. As the new behaviour is learned or incorporated into the daily routine these rewards are gradually reduced from daily to weekly and then eliminated completely.

For adolescents and adults self-reward is an alternative to reinforcement given by others. Self-reward is important, as extrinsic rewards can lose their influence over time for promoting self-care (Enzle et al. 1991). As with reinforcement delivered by others the rewards selected need to be appropriate to the behaviour change and individualized to the patient. Identification of several rewards that can be used interchangeably is instrumental in preventing boredom. It is also important to specify what behaviour needs to change and how much change is necessary to earn the reward. Again, it is more effective if patients reward themselves for small changes that gradually lead to larger changes. Rewards should be delivered after the behaviour change, not before, and rewards should be delivered close in time to the accomplished behaviour change (immediacy).

\section{Contracting}

Formalizing the systematic use of contingency management through the use of contracting is useful (Miller \& Stark, 1994). Contracting is a written agreement that defines the targeted behaviour, the consequences that will be provided contingent upon the occurrence of the targeted behaviour, and who will provide the consequences (La Greca \& Schuman, 1995). Contracts can be formalized between patients and their parents and/or patients and health care providers. Initially, Be In CHARGE! uses contracts between the children and group leaders. A contract is signed each week in which the children agree to 'get more energy' at the target meal and, in exchange, the group leaders award a trophy for achieving this goal. The contracts are simple statements of this contingency and are designed with a colourful picture of a trophy. As the treatment progresses, contracts are used to define the home-based privileges the parents will provide when the children meet their daily and weekly 'energy' goal. Contracts are useful in decreasing conflict between a child and parent because they explicitly define the behavioural expectation and rewards. They may also enhance communication skills between health care providers, patients and parents because the expected behaviour must be defined as well as the consequence for the behaviour. Finally, contract negotiation and follow-through may have additional benefits of enhancing self-control and development of adaptive healthy behaviours (Miller \& Stark, 1994).

\section{Summary}

Behavioural interventions have been found to be useful in modifying dietary behaviour. Many of the structural aspects of behavioural interventions can be readily adapted to nutritional counselling. These aspects include self-monitoring and feedback and setting realistic goals that gradually increase over time. Dietary changes are typically lifelong behaviour changes that can be complex. Additional behavioural techniques may be necessary to effect change, and may include identifying environmental barriers and introducing a way of overcoming these barriers through reinforcement of the dietary behaviour change. For children, this intervention most often necessitates changing the interaction between parent and child to include differential attention, contingency management and contracting.

\section{Acknowledgments}

Support for preparation of this paper was provided by grant K24 DK59492 from the National Institutes of Health to the author.

\section{References}

Anthony H, Paxton S, Bines J \& Phelan P (1999) Psychosocial predictors of adherence to nutritional recommendations and growth outcomes in children with cystic fibrosis. Journal of Psychosomatic Research 47, 623-634.

Beker LT, Russek-Cohen E \& Fink RJ (2001) Stature as a prognostic factor in cystic fibrosis survival. Journal of the American Dietetic Association 101, 438-442. 
Bell L, Durie P \& Forstner GG (1984) What do children with cystic fibrosis eat? Journal of Pediatric Gastroenterology and Nutrition 3, Suppl. 1, S137-S146.

Birch LL (1990) Development of food acceptance patterns. Developmental Psychology 26, 515-519.

Borowitz D, Baker RD \& Stallings VA (2002) Consensus report on nutrition for pediatric patients with cystic fibrosis. Journal of Pediatric Gastroenterology and Nutrition 35, 246-259.

Bowen AM \& Stark LJ (1991) Malnutrition in cystic fibrosis: A behavioral conceptualization of cause and treatment. Clinical Psychology Review 11, 315-331.

Chase HP, Long MA \& Lavin MH (1979) Cystic fibrosis and malnutrition. Journal of Pediatrics 95, 337-347.

Corey M \& Farewell V (1996) Determinants of mortality from cystic fibrosis in Canada. American Journal of Epidemiology 143, 1007-1017.

Corey M, McLaughlin FJ, Williams M \& Levison H (1988) A comparison of survival, growth, and pulmonary function in patients with cystic fibrosis in Boston and Toronto. Journal of Clinical Epidemiology 41, 583-591.

Crist W, McDonnell P, Beck M, Gillespie CT, Barrett P \& Mathews J (1994) Behavior at mealtimes and nutritional intake in the young child with cystic fibrosis. Developmental and Behavioral Pediatrics 15, 157-161.

Crozier DN (1974) Cystic fibrosis - a not so fatal disease. Pediatric Clinics of North America 21, 935-950.

Cystic Fibrosis Foundation (2002) Patient Registry 2001 Annual Data Report. Bethesda, MD: Cystic Fibrosis Foundation.

Daniels L, Davidson GP \& Martin AJ (1987) Comparison of the macronutrient intake of healthy controls and children with cystic fibrosis on low fat or nonrestricted fat diets. Journal of Pediatric Gastroenterology and Nutrition 6, 381-386.

Eddy MD, Carter BD, Kronenberger WG, Conradsen S, Eid NS, Bourland SL \& Adams G (1998) Parent relationships and compliance in cystic fibrosis. Journal of Pediatric Health Care 12, 196-202.

Enzle ME, Roggeveen JP \& Cook SC (1991) Self versus other reward administration and intrinsic motivation. Journal of Experimental Psychology 27, 468-479.

Epstein LH, Wing RR, Steranchak L, Dickson B \& Michelson J (1980) Comparison of family-based behavior modification and nutrition education for childhood obesity. Journal of Pediatric Psychology 5, 25-35.

Forehand RL \& McMahon RJ (1981) Helping the Noncompliant Child. New York: The Guilford Press.

Henley LD \& Hill ID (1990) Errors, gaps, and misconceptions in the disease-related knowledge of cystic fibrosis patients and their families. Pediatrics 85, 1008-1014.

La Greca AM \& Schuman WB (1995) Adherence to prescribed medical regimens. In Handbook of Pediatric Psychology, 2nd ed., pp. 55-83 [MC Roberts, editor]. New York: The Guilford Press.

Linscheid TR (1983) Eating problems in children. In Handbook of Clinical Child Psychology, pp. 616-639 [CE Walker and MC Roberts, editors]. New York: John Wiley \& Sons.

Linscheid TR, Budd KS \& Rasnake LK (1995) Pediatric feeding disorders. In Handbook of Pediatric Psychology, 2nd ed., pp. 501-515 [M Roberts, editor]. New York: The Guilford Press.

Luder E, Kattan M, Thorton JC, Koehler KM \& Bonforte RJ (1989) Efficacy of a nonrestricted fat diet in patients with cystic fibrosis. American Journal of Diseases of Children 143, 458-464.

Meichenbaum D \& Turk D (editors) (1987) Facilitating Treatment Adherence: A Practitioner's Guidebook. New York NY: Plenum Press.

Miller DL \& Stark LJ (1994) Contingency contracting for improving adherence in pediatric populations. Journal of the American Medical Association 271, 81-83.
Powers SW, Patton SR, Byars KC, Mitchell MJ, Jelalian E, Mulvihill MM, Hovell MF \& Stark LJ (2002) Caloric intake and eating behavior in infants and toddlers with cystic fibrosis. Pediatrics 109, 1-10.

Ramsey B, Farrell P \& Pencharz P (1992) Nutritional assessment and management in cystic fibrosis: Consensus conference. American Journal of Clinical Nutrition 55, 108-116.

Ryan P \& Lauver DR (2002) The efficacy of tailored interventions. Journal of Nursing Scholarship 34, 331-337.

Sanders MR, Turner KM, Wall CR, Waugh LM \& Tully LA (1997) Mealtime behavior and parent-child interaction: A comparison of children with cystic fibrosis, feeding problems, and nonclinic controls. Journal of Pediatric Psychology 22, 881-900.

Satter E (1987) How to Get Your Child to Eat, But Not Too Much. Palo Alto, CA: Bull Publishing Company.

Smith AE \& Lloyd-Still JD (1983) Value of computerized dietary analysis in pediatric nutrition: An analysis of 147 patients. Journal of Pediatrics 103, 820-824.

Spieth L, Stark LJ, Mitchell MJ, Schiller M, Cohen LL, Mulvihill MM \& Hovell M (2001) Observational assessment of family functioning at mealtime in preschool children with cystic fibrosis. Journal of Pediatric Psychology 26, 215-224.

Stark LJ, Bowen AM, Tyc VL, Evans S \& Passero MA (1990) A behavioral approach to increasing calorie consumption in children with cystic fibrosis. Journal of Pediatric Psychology 15, 309-326.

Stark LJ, Jelalian E, Mulvihill MM, Powers SW, Bowen AM, Spieth LE, Keating K, Evans S, Creveling S, Harwood I, Passero MA \& Hovell MF (1995) Eating in preschool children with cystic fibrosis and healthy peers: A behavioral analysis. Pediatrics 95, 210-215.

Stark LJ, Jelalian E, Powers SW, Mulvihill MM, Opipari LC, Bowen A, Harwood I, Passero MA, Lapey A, Light M \& Hovell MF (2000) Parent and child mealtime behaviors in families of children with cystic fibrosis. Journal of Pediatrics 136, 195-200.

Stark LJ, Knapp L, Bowen AM, Powers SW, Jelalian E, Evans S, Passero MA, Mulvihill MM \& Hovell M (1993) Increasing calorie consumption in children with cystic fibrosis: Replication with two year follow-up. Journal of Applied Behavior Analysis 26, 435-450.

Stark LJ, Mulvihill MA, Powers SW, Jelalian E, Keating K, Creveling S, Brynes-Collins B, Miller DL, Harwood I, Passero MA, Light M \& Hovell M (1996) Behavioral intervention to improve calorie intake of children with cystic fibrosis: Treatment vs. wait-list controls. Journal of Pediatric Gastroenterology and Nutrition 22, 240-253.

Stark LJ, Mulvihill MM, Jelalian E, Bowen AM, Powers SW, Tao S, Creveling S, Passero MA, Harwood I, Light M, Lapey A \& Hovell MF (1997) Descriptive analysis of eating behavior in school-age children with cystic fibrosis and healthy control children. Pediatrics 99, 665-671.

Stark LJ, Opipari LC, Spieth L, Jelalian E, Quittner AL, Higgins L, Mackner LM, Byars KC, Lapey A, Stallings VA \& Duggan C (2003) Contribution of behavior therapy to dietary treatment in cystic fibrosis: A randomized controlled study with two-year follow-up. Behavior Therapy 34, 237-258.

Tomezsko JL, Stallings VA \& Scanlin TF (1992) Dietary intake of healthy children with cystic fibrosis campared with normal control children. Pediatrics 90, 547-553.

Zemel BS, Jawad AF, FitzSimmons S \& Stallings VA (2000) Longitudinal relationship among growth, nutritional status and pulmonary functioning in children with cystic fibrosis: analysis of the Cystic Fibrosis Foundation National CF Patient Registry. Journal of Pediatrics 137, 374-380. 